\title{
Dust charge distribution in complex plasma afterglow
}

\author{
L. Couëdel ${ }^{1,2},{ }^{*}$ A.A. Samarian ${ }^{1}$, M. Mikikian ${ }^{2}$, and L. Boufendi ${ }^{2}$ \\ 1 School of Physics A28, The University of Sydney, NSW 2006, AUSTRALIA \\ ${ }^{2}$ GREMI (Groupe de Recherches sur l'Énergétique des Milieux Ionisés), \\ CNRS/Université d'Orléans, 14 rue d'Issoudun, 45067 Orléans Cedex 2, FRANCE
}

(Dated: July 1, 2008)

\begin{abstract}
Dust charge distributions have been measured for the first time in a complex plasma. Experiments were performed late in a discharge afterglow. Residual charges of few hundred particles have been determined. It was found that the mean residual charge is negative but the tail of the distribution extends into the positive charge region. The experiments were complemented by numerical simulations taking into account discreteness of the charging process and dependence of plasma parameters on the transition from ambipolar to free diffusion. It is shown that the existence of positively charged particles can be explained by the broadening of the dust charge distribution in the late complex plasma afterglow.
\end{abstract}

PACS numbers: 52.27.Lw

Complex (or dusty) plasmas are partially ionized gases composed of neutral species, ions, electrons and charged dust particles. They are very common and can be either natural plasmas (such as near-Earth plasmas (in which the sources of dust are meteor combustion material, volcanoes, dust storms, aerosol pollution, etc), comet tail plasmas, planetary rings) as well as human-made plasmas (etching, PECVD, fusion reactors, etc) [1, 2].

In most space and laboratory plasmas, dust particles are charged. The charging of a dust particle is mainly due to the current of ions and electrons onto the dust particle surface [1]. This leads to unusual phenomena in complex plasma systems $[3,4]$ and distinguishes them from classical gas plasmas. In an uniform steady state plasma ion and electron currents compensate each other and dust particles acquire an equilibrium charge. However due to the discretness of the charge carriers, the charging process is stochastic and dust charge fluctuations occur.

Dust charge and its fluctuation are key factors for many complex plasma problems ranging from basic phenomena like interparticle interactions and shielding to selforganisation effects in dust cloud dynamics and the formation of complex electrostatically coupled systems. Thus one of the basic problems is the determination of the dust charge distribution (DCD). A clear understanding of DCDs is of interest when dealing with layer depositions (where charged dust particles strongly lower the quality of the deposited films in microelectronics for example), when dealing with nanoparticle deposition for nanostructured materials or single electron devices in nanotechnologies. DCDs are also of interest in aerosol physics where solid particles influence their environment depending on their electric charges (for example fog [5]). Furthermore measurement of residual dust charges opens a unique opportunity for afterglow plasma diagnostics (for example in pulsed plasma sources widely used in laboratory and technological plasmas).

Charge fluctuations and DCDs have been the subject of many theoretical studies ([6] and references therein). In particular, Matsoukas et al $[7,8]$ reported a DCD obtained using a Fokker-Planck model of dust charging (discretness of the charging process). A similar result was obtained by Tsytovich et al [6] assuming the discretness of the electron and ion distributions. Khrapak et al [9] obtained the magnitude of fluctuation in terms of the temporal autocorrelation function and reported dependence of the DCD on plasma parameters. All proposed models confirm that the DCD is Gaussian and that the RMS of the stochastic charge fluctuation varies as $\sigma\left(Q_{d}\right) \simeq \delta \sqrt{\left|\bar{Q}_{d}\right|}$, where $Q_{d}$ is the dust particle charge, $\bar{Q}_{d}$ is the mean dust particle charge and $\delta$ is a parameter depending on plasma conditions and is close to 0.5. Computer simulations of the charging process also report the same result [10-13]. Nevertheless, no direct measurement of the DCD has been performed due to the shortness of the charging and characteristic charge fluctuation timescales (in the $\mu s$ range). So at any particular moment an ensemble of monosized dust particle is characterized by its DCD but on the dust time scale (in the $m s$ range) the dust behavior is determined by its mean equilibrium charge. Existing experimental techniques for dust charge measurement based on the analysis of dust dynamic response [14-16] can only determine the mean charge which is equal for all monosized particles. Complex plasma afterglow provides a unique opportunity to perform measurements of the DCD. In a decaying complex plasma, the dust particle charges as well as the DCD evolve with the other plasma parameters such as the plasma density and the electron temperature. In the late stage of plasma decay the dust charge is frozen and does not change further. The DCD remains unchanged for a long time. It has been shown that in the late afterglow of a complex plasma the dust particles do keep a residual charge for a long time (few seconds) [17]. It allows the measurements of dust charges and DCDs. The coexistence of positively charged, negatively charged and neutral dust particles in the late afterglow was observed in [18], however no dust charge distribution has been re- 
ported and no explanation for the presence of positively charged particles has been given.

In this letter, we report the first experimental measurements of the DCD in the late stage of a complex plasma afterglow. It has been found that the mean residual charge is negative and the DCD spreads into the positive charge region. A model taking into account the dependence of plasma parameters on a transition from ambipolar to free diffusion for the ions and the electrons is used to explain the observed DCD.

The experimental residual charge distributions have been obtained using the PKE-Nefedov reactor [19]. It is a RF discharge operating in push-pull excitation mode consisting of $4 \mathrm{~cm}$ diameter parallel electrodes separated by 3 $\mathrm{cm}$ (see Fig.1). The injected power varies in the range $0-4 W$. Dust particles are grown in an argon plasma $(0.2-2$ mbar $)$ from a sputtered polymer layer deposited on the electrodes which comes from previously injected dust particles (3.4 $\mu \mathrm{m}$, melamine formaldehyde) [20].

The charge on dust particles has been measured by analyzing the dust dynamic response to an external electric field (pulsed, constant, sinusoidal). The best results were obtained for a sinusoidal electric field. The sinusoidal voltage was produced by a function generator with amplitude $\pm 30 \mathrm{~V}$ and frequency of $f=1 \mathrm{~Hz}$, applied to the bottom electrode. The induced low frequency sinusoidal electric field generated oscillations of the dust particles (Fig.1). A thin laser sheet perpendicular to the electrodes illuminated the dust particles and the scattered light was recorded at $90^{\circ}$ with standard charge coupled device (CCD) cameras (25 images per second). By superimposing video frames and scaling the picture, the particle oscillatory trajectories have been constructed (see image in Fig.1). In order to get enough statistics to construct representative DCDs, the dust particles were levitated in the discharge afterglow using thermophoresis by cooling down the top electrode [21]. The temperature gradient was adjusted to levitate $\sim 200 \mathrm{~nm}$ radius particles.

The charge on a dust particle $Q_{d}$ can be obtained from the oscillation amplitude $b[18]$ :

$$
Q_{d_{r e s}}=\left(m_{d} b \omega \sqrt{\omega^{2}+4 \gamma^{2} / m_{d}^{2}}\right) / E
$$

where $m_{d}$ is the mass of the dust particle, $E$ and $\omega$ are the amplitude and angular frequency of the sinusoidal electric field respectively and $\gamma$ is the damping coefficient. The sets of dust particle oscillation amplitudes were determined by analyzing particle trajectories. By measuring oscillations of many dust particles, dust oscillation amplitude distributions have been constructed and were converted to DCDs (Eq.1). The sign of the dust particle charge was deduced from the phase of the dust particle oscillation with respect to the electric field. Taking into account all sources of errors (radius, mass, electric field, neutral drag force), the residual charge on each particle
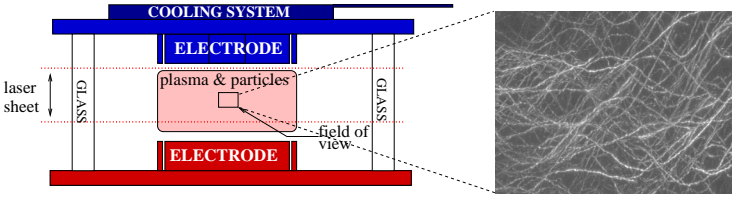

FIG. 1: Left: Schematic of the PKE-Nefedov reactor. Right: Superimposition of video frames
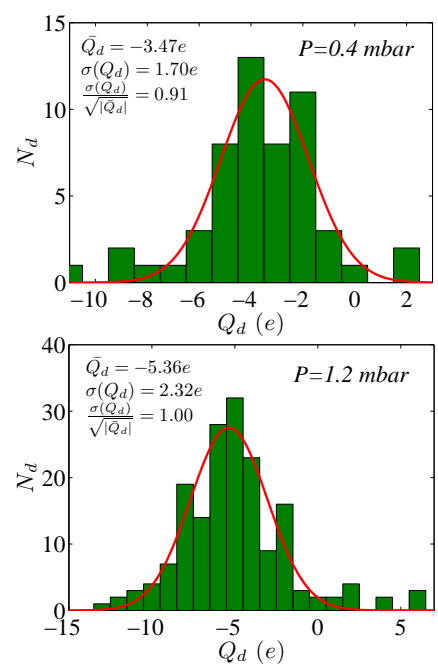

FIG. 2: (Color online) Experimental DCD. Top: $P=$ 0.4 mbar; bottom $P=1.2$ mbar

is known with a precision of $\pm 49 \%$.

The resulting discharge afterglow DCDs are presented in Fig.2 for two operating pressures. Both distributions have been fitted by a Gaussian function (red line). The mean residual charges are negative for both pressures and their values correspond to a few electrons $\left(\bar{Q}_{D_{\text {res }}} \sim-3 e\right)$ for 0.4 mbar and $\left(\bar{Q}_{D_{\text {res }}} \sim-5 e\right)$ for 1.2 mbar. Positive residual charges were observed for the both cases. The RMS of the DCD is of the same order of magnitude for both pressures $(\sigma \sim 2 e)$, see Fig.2. The coefficient $\delta=\sigma\left(Q_{d}\right) / \sqrt{\left|\bar{Q}_{d}\right|}$ is about unity. The accuracy on the charge measurement artificially enlarges the experimental DCD: for 0.4 mbar, $\delta=0.91 \pm 0.09$ has been measured but can correspond to an estimated error-free width of $\delta=0.72 \pm 0.11 ;$ for $1.2 \operatorname{mbar} \delta=1 \pm 0.07$ has been measured but can correspond to an estimated error-free width of $\delta=0.79 \pm 0.09$. In both cases the estimated values of $\delta$ are bigger than the $\delta=0.5$ predicted for a running discharge $[7-9,11,12]$.

The existence of residual charge was explained in Ref.[17]]: the residual charges were attributed to electron cooling and the frozen stage was achieved when the charging time becomes much longer than the diffusion time. However the model predicted only the value of the mean rest charge. In Ref. [18], a more detailed model was proposed. The model includes four stages: Firstly, the electron temperature decreases to room temperature 
while the dust particle charge decreases drastically (in absolute value), then the plasma diffuses ambipolarly until the Debye length reaches the diffusion length and the dust charge remains almost constant. Next, the ions and the electrons diffuse freely and due to the higher mobility of electrons, soon only ions remain in the discharge. This leads to a small decrease of the dust particle charge. Finally, the plasma density is such that the absorption time of electrons and ions becomes much longer than the diffusion time which forbids any further change of the dust particle charge which will not fluctuate measurably. Consequently, any measurements of DCDs made in the very late afterglow are representative of the frozen DCDs. Nevertheless, this model also predicts the mean charge only and is unable to explain the existence of the positively charged as well as the neutral dust particles.

In order to understand experimental DCDs, simulation of a complex plasma afterglow has been done. A numerical model taking into account plasma decay as well as dust charge evolution in discharge afterglow has been developed. Temperature relaxation and plasma losses due to diffusion have been treated using the equations of Ref.[18]. The dust particle charges, as well as the plasma losses due to recombination onto the surface of dust particles, were computed using the Fokker-Planck description of dust charging. The dust charge varied in steps of $\pm e$ with the probability per unit of time given by electron and ion currents on the dust surface per absolute value of the electron charge [11].

In Refs. $[17,18]$ diffusion losses were attributed to ambipolar diffusion at the first stage and to the free diffusion at later stages (after the Debye screening length exceeds the diffusion length). Here the diffusion losses were computed taking into account experimental data on the transition from ambipolar to free diffusion [22, 23]. These data give the ratio of ion and electron diffusion coefficients to the ambipolar diffusion coefficient $D_{a}$ as a function of the ratio $\left(\Lambda / \lambda_{D e}\right)^{2}$ in helium plasma where $\lambda_{D e}$ is the electron Debye length. The ambipolar diffusion coefficient is:

$$
D_{a} \propto\left(\left(k_{B} T_{i}\right)^{3 / 2}\right) /\left(p \sigma_{i n} m_{i}^{1 / 2}\right)
$$

where $k_{B}$ is the Boltzmann constant, $T_{i}$ is the ion temperature, $m_{i}$ is the ion mass, $p$ is the neutral gas pressure and $\sigma_{i n}$ is the ion-neutral collision cross section. As $\sigma_{i n_{A r^{+}}} \sim 2.5 \sigma_{i n_{\mathrm{He}^{+}}}[24]$ and $m_{\mathrm{Ar}^{+}} \sim 10 m_{i_{\mathrm{He}}+}$, $D_{a_{A r}} \sim(1 / 8) \cdot D_{a_{H e}}$ for equal pressures. Consequently, it can be assumed that the diffusion of an argon plasma with argon pressure $p_{A r}$ has the same behaviour as an helium plasma with helium pressure $p_{H e} \simeq 8 \cdot p_{A r}$. The available experimental data for Helium are in the range of pressure 0.4-4 Torr [22] and 9-22.8 Torr [23] which correspond to $0.05-0.5$ Torr and $1.13-2.85$ Torr respectively in equivalent argon pressure. Our simulations were performed in the range $0.3-1$ Torr (0.4 - 1.3 mbar) Consequently, the data from Ref.[22]
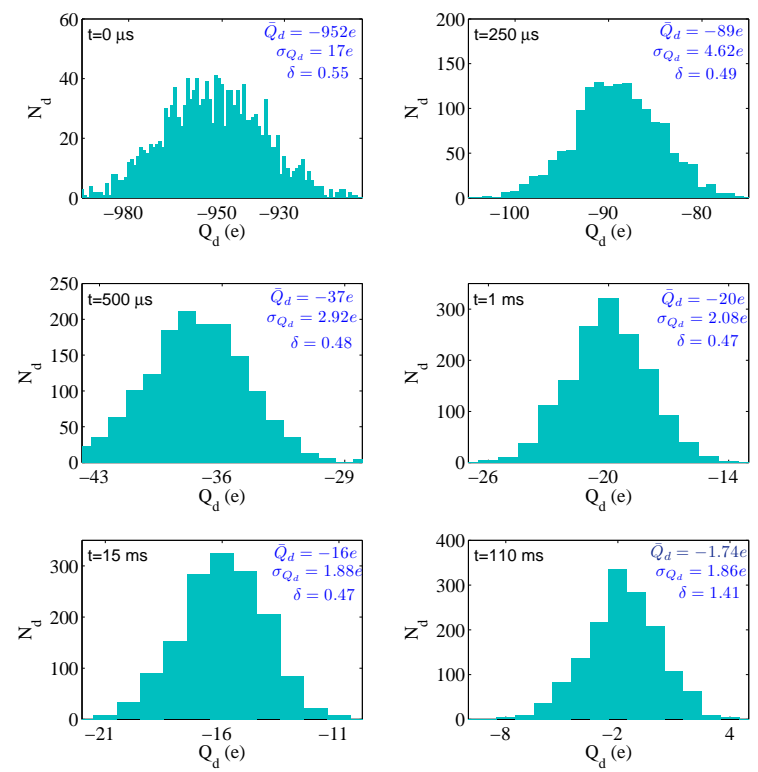

FIG. 3: (Color online) Simulated DCDs at different times in the afterglow plasma $\left(P=1.2 \mathrm{mbar}\right.$ and $\left.\sum N_{d}=1500\right)$.

are used to compute the diffusion time of electrons and data from Ref.[23] are used for ions as they are only data available. The initial ion density was $n_{i 0}=5 \cdot 10^{9} \mathrm{~cm}^{-3}$ and the dust density is $n_{d}=5 \cdot 10^{4} \mathrm{~cm}^{-3}$. The DCD was computed using a modified algorithm reported in Ref. [11]. The initial DCD was obtained using the quasi-neutrality condition: $Z_{d} n_{d}+n_{e}=n_{i}$ where $Z_{d}=\left|Q_{d} / e\right|$. The initial temperatures are $T_{e}=3 \mathrm{eV}$ and $T_{i}=T_{n}=0.03 \mathrm{eV}$ where $T_{n}$ is the gas temperature. The initial electron density $n_{e 0}$ is deduced from this calculation. The obtained DCD is presented in Fig.3 $(t=0 \mu s)$. The mean charge is $\bar{Q}_{d}=-952 e$ and the variance $\sigma\left(Q_{d}\right)=17 e$ leading to $\delta=\sigma\left(Q_{d}\right) / \sqrt{\left|\bar{Q}_{d}\right|}=0.55$. In Fig.3, the simulated DCDs at different time in the plasma afterglow are presented. The evolution of dust $\bar{Q}_{d}$ and $\delta$ in the afterglow are shown in Fig.4(a) and Fig.4(b) respectively. The obtained mean dust charge evolution is in agreement with qualitative predictions of the four stage model (Fig.7 in Ref.[18]).

During the first stage of the plasma decay, the charging time of dust particles $t_{Q_{0}}$ is very short compared to the plasma loss time $\tau_{L}$ (typically $t_{Q_{0}} \sim 1-10 \mu \mathrm{s}<<\tau_{L} \sim 1-10 \mathrm{~ms}$ ) so the charge on dust particles can be considered to be in equilibrium with the surrounding plasma. The electron temperature relaxes to the room temperature leading to a strong decrease of the dust charge. In Figs.4(a) and 3, this corresponds to the first millisecond. Then during the plasma decay stage dust charge stabilizes. During the third stage charge decreases until it becomes frozen. The simulation is stopped during the fourth stage when the charging time becomes much bigger than the diffusion 

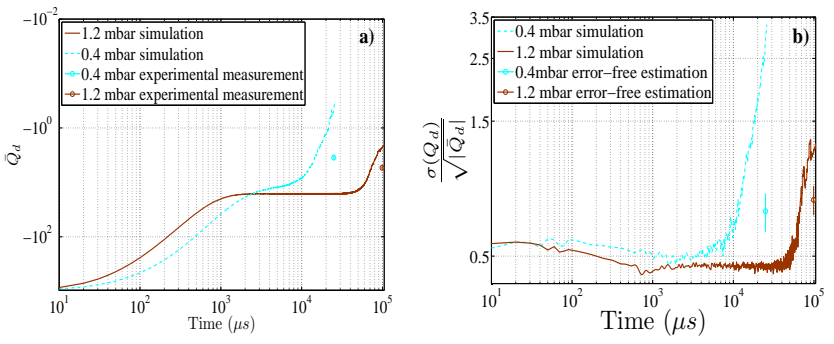

FIG. 4: (Color online) a) Evolution of the mean charge $\bar{Q}_{d}$ in the afterglow plasma. b) Evolution of the ratio $\delta=$ $\sigma\left(Q_{d}\right) / \sqrt{\mid \overline{Q_{d} \mid}}$ in the afterglow plasma. Experimental results have been placed near their corresponding simulation curves; these measurements have been performed a few seconds after the discharge was switched off

time.

As can be seen in Fig.4(b), $\delta$ decreases slightly in the first two stages down to $\delta \sim 0.47$. This value agrees with theoretical prediction of Khrapak et al. which gives $\delta=0.50$ for $T_{e} / T_{i}=20$ and $\delta=0.46$ for $T_{e} / T_{i}=1$ in an argon plasma [9]. Drastic DCD broadening is observed in the third stage when the charge of the particles continues to decrease $(t=15 \mathrm{~ms}$ in Fig.3) and $\delta$ is steeply increasing ( Figs.4(a) and 4(b)). The broadening of the distribution was observed until the end of the simulation. As can be seen in Fig.3 $(t=110 \mathrm{~ms})$, the frozen DCD is very wide with a tail in the positive charge region. The simulated DCD is in good agreement with the experimental one (Fig.2). However as can be seen in Figs.4(a) and 4(b), simulations do not reproduce exactly the observed values of $\bar{Q}_{d}$ and $\delta$. This discrepancy can be explained by the strong influence of the character of diffusion losses on dust charging in the afterglow. Simulation shows that the transition from ambipolar to free diffusion plays a major role in DCD evolution. In our calculation we use data from [22] which may not be accurate enough for good agreement between experimental and simulated DCD. Additional investigation of the effect of the transition from ambipolar to free diffusion on the dust charging in an afterglow plasma is so required and is the subject of current studies.

To conclude, the first measurement of the DCD in a complex plasma afterglow has been reported. The mean dust particle residual charge is $\bar{Q}_{d_{\text {res }}} \sim-4 e$. It was found that the DCD in the plasma afterglow is much broader than the one expected in a steady discharge $\left(\delta_{a f t} \sim 0.75>\delta_{p l} \sim 0.5\right)$, allowing the tail of the DCD to extend in positive range values. This fact explains the existence of positively charged particles in an afterglow plasma. Numerical simulation of the complex plasma afterglow showed that the width of the DCD strongly depends on the transition from the ambipolar to free diffusion.

The authors would like to thank S. Dozias and B.
Dumax for electronic support, J. Mathias for optical support, Y. Tessier, C. Cuthbert and J. Sisovic for experimental support. The PKE-Nefedov chamber has been made available by the MPE, Germany, under the funding of DLR/BMBF under grants No.50WM9852. This work was supported by CNES under contract 793/2000/CNES/8344. This paper is partially supported by the French-Australian integrated research program (FAST) under contract No.FR060169.

*lectronic address: lenaic.couedel@univ-orleans.fr

[1] S. Vladimirov, K. Ostrikov, and A. Samarian, Physics and Applications of Complex Plasmas (Imperial Press, London, 2005).

[2] A. Bouchoule, Dusty Plasmas: Physics, Chemistry and Technological impacts in Plasma Processing (Wiley, New York, 1999).

[3] M. Mikikian, L. Couëdel, M. Cavarroc, Y. Tessier, and L. Boufendi, New J. Phys. 9, 268 (2007).

[4] M. Cavarroc, M. Mikikian, G. Perrier, and L. Boufendi, Appl. Phys. Lett. 89, 013107 (2006).

[5] M. Anisimov, M. Mareev, G. Sorokin, and L. Dmitriev, Atmospheric Res. 76, 16 (2005).

[6] G. E. Morfill, V. N. Tsytovich, and H. Thomas, Plasma Phys. Rep. 29, 1 (2003).

[7] T. Matsoukas and M. Russell, J. Appl. Phys. 77, 4285 (1995).

[8] T. Matsoukas, M. Russell, and M. Smith, J. Vac. Sci. Technol. A 14, 624 (1996)

[9] S. A. Khrapak, A. P. Nefedov, O. F. Petrov, and O. S. Vaulina, Phys. Rev. E 59, 6017 (1999).

[10] T. K. Alaksen, J. Plasma Phys. 54, 373 (1995).

[11] C. Cui and J. Goree, IEEE Trans. Plasma Sci. 22, 151 (1994).

[12] O. S. Vaulina, S. A. Khrapak, A. P. Nefedov, and O. F. Petrov, Phys. Rev. E 60, 5959 (1999).

[13] S. A. Maiorov, S. V. Vladimirov, and N. F. Cramer, Plasma Phys. Rep. 28, 946 (2002).

[14] C. Arnas, M. Mikikian, and F. Doveil, Phys. Scripta T89, 163 (2001).

[15] A. A. Samarian and S. V. Vladimirov, Phys. Rev. E 67, 066404 (2003).

[16] A. Melzer, T. Trottenberg, and A. Piel, Phys. Lett. A 191, 301 (1994).

[17] A. Ivlev, et al., Phys. Rev. Lett. 90, 055003 (2003).

[18] L. Couëdel, M. Mikikian, L. Boufendi, and A. A. Samarian, Phys. Rev. E 74, 026403 (2006).

[19] A. P. Nefedov, G. E. Morfill, V. E. Fortov, H. M. Thomas, et al., New J. Phys. 5, 33 (2003).

[20] M. Mikikian, M. Cavarroc, L. Couëdel, et al., Phys. Plasmas 13, 092103 (2006).

[21] H. Rothermel, T. Hagl, G. E. Morfill, M. H. Thoma, and H. M. Thomas, Phys. Rev. Lett. 89, 175001 (2002).

[22] R. J. Freiberg and L. A. Weaver, Phys. Rev. 170, 336 (1968).

[23] R. A. Gerber and J. B. Gerardo, Phys. Rev. A 7, 781 (1973).

[24] R. N. Varney, Phys. Rev. 88, 362 (1952). 